\title{
Endovascular Aortic Aneurysm and Dissection Repair(EVAR) in Iran: Descriptive Midterm Follow-up Results
}

\author{
Ali Mohammad Haji Zeinali, ${ }^{1, *}$ Mehrab Marzban, ${ }^{2}$ Mohammadreza Zafarghandi, ${ }^{3}$ Mahmood \\ Shirzad, ${ }^{2}$ Shapour Shirani, ${ }^{4}$ Roshanak Mahmoodian, ${ }^{5}$ Mehrdad Sheikhvatan, ${ }^{5}$ and Ma- \\ soumeh Lotfi-Tokaldany ${ }^{5}$ \\ ${ }_{2}^{1}$ Department of Interventional Cardiology, Tehran Heart Center, Tehran University of Medical Sciences, Tehran, Iran \\ ${ }_{3}^{2}$ Department of Cardiac Surgery, Tehran Heart Center, Tehran University of Medical Sciences, Tehran, Iran \\ ${ }_{4}^{3}$ Department of Vascular Surgery, Sina Hospital, Tehran University of Medical Sciences, Tehran, Iran \\ ${ }_{5}^{4}$ Department of Radiology, Tehran Heart Center, Tehran University of Medical Sciences, Tehran, Iran \\ ${ }^{5}$ Department of Research, Tehran Heart Center, Tehran University of Medical Sciences, Tehran, Iran \\ *Corresponding author: Ali Mohammad Haji Zeinali, Department of Interventional Cardiology, Tehran Heart Center, Tehran University of Medical Sciences, P. O. Box: 1411713138, Tehran, Iran. \\ Tel:+98-218802960060, Fax:+98-2188029731, E-mail:ali_zeinali_cardio@yahoo.com
}

Received: November 5, 2013; Revised: July 12, 2014; Accepted: August 25, 2014

\begin{abstract}
Background: Endovascular repair of aorta in comparison to open surgery has a low early operative mortality rate, but its long-term results are uncertain.

Objectives: The current study describes for the first time our initial four-year experience of elective endovascular aortic repair (EVAR) at Tehran heart center, the first and a major referral heart center in Iran, as a pioneer of EVAR in Iran.

Patients and Methods: $A$ total of 51 patients (46 men) who had the diagnosis of either an abdominal aortic aneurysm (AAA) ( $\mathrm{n}=36)$, thoracic aortic aneurysm (TAA) $(n=7)$, or thoracic aortic dissection $(T A D)(n=8)$ who had undergone EVAR by Medtronic stent grafts by our team between December 2006 and June 2009 were reviewed.

Results: The rate of in-hospital aneurysm-related deaths in the group with AAA stood at 2.8\% (one case), while there was no in-hospital mortality in the other groups. All patients were followed up for 13-18 months. The cumulative death rate in follow-up was nine cases from the total 51 cases (18\%), out of which six cases were in the AAA group (four patients due to non-cardiac causes and two patients due to aneurysmrelated causes), one case in the TAA group (following a severe hemoptysis), and two cases in the TAD group (following an expansion of dissection from re-entrance). The major event-free survival rate was $80.7 \%$ for endovascular repair of AAA, $85.7 \%$ for endovascular repair of TAA, and $65.6 \%$ for endovascular repair of TAD.

Conclusion: The endovascular stent-graft repair of the abdominal and thoracic aortic aneurysm and aortic dissection had high technical success rates in tandem with low-rate early mortality and morbidity, short hospital stay, and acceptable mid-term free symptom survival among Iranian patients.
\end{abstract}

Keywords: Endovascular Procedure; Aortic Diseases; Aortic Aneurysm; Dissecting; Iran

\section{Background}

Despite the acceptability of open surgical repair in patients with aortic aneurysm or dissection, particularly for those considered fit enough to withstand major surgery, the survival rate of the patients undergoing open surgery remains low (1). In the recent decade, the availability of new devices and enhancement of the quality of procedural capabilities have ushered improvement in the treatment outcome and reduction of the rates of morbidity and mortality $(2,3)$.

Endovascular therapy and stent-graft interventions are exciting new frontiers in the treatment of aortic disorders and they can be effectively achieved with high technical success rates and confer favorable survival rates $(4,5)$. These minimally invasive approaches have considerable perioperative advantages such as reduced blood loss, reduced stay in the intensive care unit, shortened hospital stay, and increased number of patients discharged to home (6). Furthermore, these procedures reduce short-term mortality rates to less than $2 \%(7,8)$. The present study describes our initial four-year experiences of elective endovascular aortic repair for the first time in Iran reported at Tehran Heart Center, a major referral heart center in Iran.

\section{Objectives}

The aim of this study was to describe the results of all first endovascular aortic repair (EVAR) experiences in Iran with midterm follow-up, to describe the safety and efficacy of this surgical alternative procedure in Iranian patients.

Copyright ( ) 2016, Tehran University of Medical Sciences and Iranian Society of Radiology. This is an open-access article distributed under the terms of the Creative Commons Attribution-NonCommercial 4.0 International License (http://creativecommons.org/licenses/by-nc/4.0/) which permits copy and redistribute the material just in noncommercial usages, provided the original work is properly cited. 


\section{Patients and Methods}

Between December 2006 and June 2009, a total of 51 patients underwent EVAR, of which 36 patients underwent endovascular abdominal aneurysm repair (mean age $=$ 69.6 years, $94.4 \%$ male), seven patients underwent endovascular thoracic aneurysm repair (mean age $=67.3$ years, $100 \%$ male), and eight patients underwent endovascular repair of the aortic dissection (mean age $=55.1$ years, 62.5\% male) at Tehran Heart Center. The demographic characteristics and co-morbidities of the patients are listed in (Table 1).

Regarding clinical manifestations, the most common symptoms in the group with abdominal aneurysm repair were abdominal pain (27.8\%), followed by abdominal mass (8.3\%) and both symptoms (2.8\%) and the remaining symptoms (61.1\%) were incidentally manifested. In the patients who experienced endovascular repair of the thoracic aorta aneurysm, one patient had an abdominal mass, two patients had chest pain, another one had hemoptysis, and the others were incidentally revealed. In the group with endovascular repair of the aortic dissection, three patients were diagnosed with subacute type B dissection with typical chest pain and five patients were diagnosed with chronic type B dissection (four cases with chest pain and another one with dysphagia).

With respect to the overall prevalence of the risk profiles, current smoking was more frequently observed in the patients undergoing thoracic aneurysm repair, while a history of hyperlipidemia and diabetes was more prevalent in those who underwent abdominal aneurysm repair. Systolic hypertension was more prevalent in the aortic dissection group than in the other groups. Previous coronary revascularization was more frequently observed in the patients who underwent abdominal aneurysm repair than in the other groups. In this case series study, all patients who had received the diagnosis of either AAA, thoracic aortic aneurysm or pseudo aneurysm, or aortic dissection who had undergone elective endovascular repair at Tehran Heart Center between December 2006 and June 2009 by our team were reviewed. The candidates were patients with abdominal aortic aneurysms with or without involvement of iliac branches, descending thoracic aortic aneurysms with or without involvement of concomitant abdominal aorta, or type B subacute or chronic dissection unsuitable for open surgery due to old age, underlying diseases, or a previous history of surgical interventions. We began EVAR based on our previous experiences on aorta interventions in our center (3, 5, 9-11).

The main indications for endovascular intervention in the aortic aneurysm were an asymptomatic abdominal aortic aneurysm diameter $>5.5 \mathrm{~cm}$ or a thoracic aortic aneurysm diameter $>5.0 \mathrm{~cm}$, enlarging at least $0.5 \mathrm{~cm}$ within six months or at least $1 \mathrm{~cm}$ within one year, or smaller symptomatic aneurysm cases. The indications for endovascular interventions in type B aortic dissection were persistent pain despite adequate pain management and critical visceral involvement. Our inclusion and exclusion criteria for case selection were based on the large United Kingdom EVAR 1 and 2 registry for AAA $(1,7)$, and VALOR trial for TAA (12) (Table 2). All of the patients underwent multislice CT angiography (256-slices CT Angiography, Siemens, Berlin, Germany) with $5 \mathrm{~mm}$ slices in our center, and current sizing was done by our expert radiologist. Then stent graft sizing and selection was performed by a team, including an interventionist, a surgeon, a radiologist and Medtronic company sizing technician (Figure 1). The Tehran heart center surgery database, operative notes, radiology records, and individual surgeon case lists were all utilized to corroborate the information. The study was approved by the institutional review board and the research committee of Tehran University of Medical Sciences.

\begin{tabular}{lccc}
\hline Table 1. Demographic Characteristics and Co-Morbidities of Patients a, b & & \\
\hline Characteristics and Risk Factors & AAA Group $(\mathbf{n}=\mathbf{3 6})$ & TAA Group $(\mathbf{n}=7)$ & TAD Group (n= 8) \\
\hline Male/Female & $34 / 2$ & $7 / 0$ & $5 / 3$ \\
Mean age, $\mathbf{y}$ & $69.61 \pm 8.31$ & $67.59 \pm 9.51$ & $55.13 \pm 14.49$ \\
Current smoking & $14(38.9)$ & $4(57.1)$ & $1(12.5)$ \\
Hypertension & $21(58.3)$ & $6(85.7)$ & $8(100)$ \\
Diabetes mellitus & $5(13.9)$ & 0 & $1(12.5)$ \\
Hyperlipidemia & $8(22.2)$ & $1(14.3)$ & $1(12.5)$ \\
Malignancy & $6(16.7)$ & 0 & 0 \\
Previous CABG & $12(33.3)$ & $1(14.3)$ & $1(12.5)$ \\
Previous PCI & $8(22.2)$ & $2(28.6)$ & $1(12.5)$ \\
\hline
\end{tabular}

a Abbreviations: AAA, abdominal aortic aneurysm; TAA, thoracic aortic aneurysm; TAD, thoracic aortic dissection; PCI, percutaneous coronary intervention.

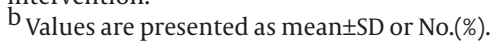


Haji Zeinali AM et al.

\begin{tabular}{ll}
\hline Table 2. Inclusion and Exclusion Criteria for EVAR and TEVAR \\
\hline Type of criteria & Criteria \\
\hline Inclusion Criteria & 1. Asymptomatic AAA $\geq 5.5 \mathrm{~cm}$ \\
\hline 2. Symptomatic AAA $\geq 3.5 \mathrm{~cm}$ \\
\hline 3. Descending thoracic AA $\geq 5 \mathrm{~cm}$ \\
\hline 4. Descending thoracic pseudoaneurysm \\
\hline 5. Subacute or chronic type B, AD with symptoms \\
\hline 6. Suitable proximal and distal neck for EVAR \\
\hline 1. Creatinine level $\geq 2 \mathrm{mg} / \mathrm{dL}$ \\
\hline 2. Patient preference for open surgery \\
\hline 3. Unsuitable peripheral arterial access \\
\hline 4. Unsuitable proximal and distal neck \\
5. Patient survival $\leq 1$ year \\
\hline
\end{tabular}

\footnotetext{
a Abbreviations: AAA, abdominal aortic aneurysm; AD, aortic dissection; AA, aortic aneurysm; TEVAR, thoracic endovascular aortic repair.
}
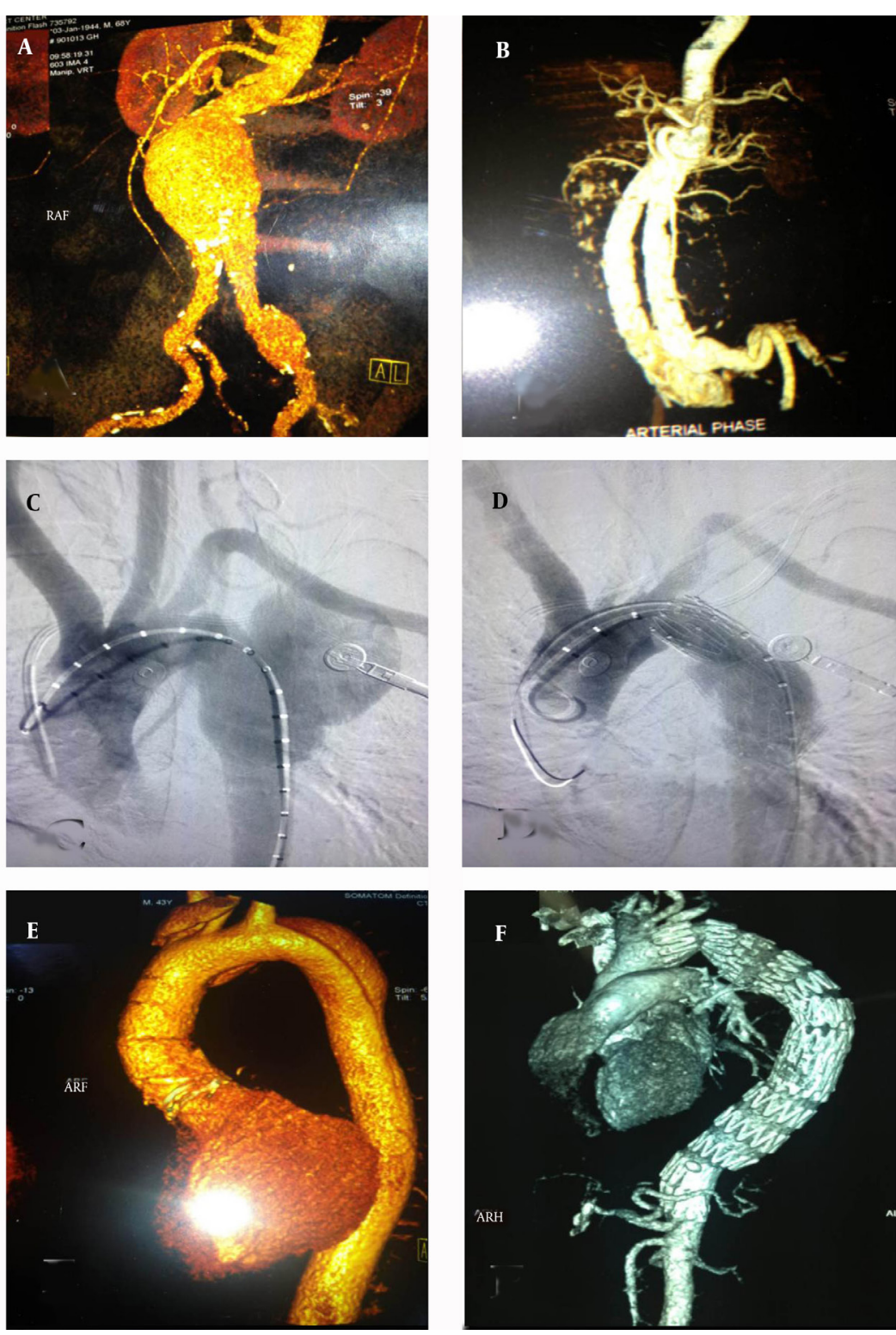

Figure 1. Pre- and post-EVAR and TEVAR images. A, AAA pre-EVAR; B, AAA post-EVAR; C, TAA pre-TEVAR; D, TAA post-TEVAR; E, AD pre-TEVAR; F, AD post-TEVAR 
For stent graft implantation, after the determination of the location and diameter of the lesion via multi-slice CT scan or angiography, the patients typically received general anesthesia and stent-grafts were inserted through the common femoral arteriotomy site and deployed once the adequate position had been confirmed under the image intensifier. All the stent grafts were talent or endorant stent-grafts (Medtronic) in the abdominal aortic aneurysms and valiant thoracic stent-grafts (Medtronic) in the thoracic aortic aneurysms or dissections.

\subsection{Statistical Analysis}

The descriptive statistics were reported as mean \pm standard deviation for the continuous variables and absolute frequencies and percentages for the categorical variables. The survival rate was measured using the KaplanMeier method.

\section{Results}

The primary outcome was the procedural success rate, inhospital mortality, post-procedural major complications, need for blood transfusion, postoperative anemia or creatinine rise, and length of hospital stay. The patients were followed up clinically and had at least one aortic imaging in the first six months with multi-slice CT angiography assessed by an experienced radiologist to evaluate the presence of endoleaks, thrombosis, change in lesion diameter, and stent migration. An endoleak refers to the persistence of the flow within the aneurysm sac despite endovascular prosthesis. Endoleaks are classified as type I-IV $(13,14)$.

The mortality and complication rates within the followup period were predetermined secondary end-points in the original trial design. The outcome events analyzed were deaths from all causes, aneurysm-related deaths, major complications, and re-interventions.

\subsection{In-Hospital and Short-Term Results of Endovas- cular Repair}

The procedural success rate was 100\%, in-hospital and one-month follow-up mortality was only one in-hospital AAA EVAR case (2.8\%), who had post procedural CVA and finally died. There were no other major complications such as MI, paraplegia, stroke, peripheral emboli, infection and others in in-hospital and short-term follow-up. Some minor complications like low-grade fever due to post implantation syndrome, mild uremia, mild anemia, and gastrointestinal symptoms were seen that were successfully managed in this period. Moreover, $11 \%$ of the patients with abdominal aneurysm repair experienced an elevation in the serum creatinine level of more than $30 \%$ over the baseline and also, a decrease in the hemoglobin level to less than $9 \mathrm{gm} / \mathrm{L}$ was found more frequently in the group with aortic dissection repair. Seven patients in the group with abdominal aneurysm repair and one patient in the group with dissection repair required blood transfusion, whereas none of the patients undergoing thoracic aneurysm repair received blood transfusion. The total lengths of in-hospital and intensive care unit stay were shorter in our AAA EVAR group than the aortic dissection TEVAR group (Table 3).

\subsection{Mid-Term Follow-Up Complications After EVAR and TEVAR}

1) In the group that underwent EVAR for AAA, endoleak type II was revealed in seven patients who were treated conservatively. Distal endoleak type I was observed in only one patient, who underwent re-stenting during some session. One patient with a previous history of chronic renal failure experienced an acute episode of renal failure with fever but negative blood culture. This patient was treated with antibiotics and did not need hemodialysis. Another heavy smoker patient developed post procedural symptoms of acute limb ischemia due to stent thrombosis with a dissecting flap. This patient was necessitated re-stenting during the same admission. He did not have any complications in follow-up. One of the patients with severe left ventricular dysfunction suffered foot paraparesis (with the final diagnosis of emboli in the lower limb) in follow-up and died finally after 6 months with the clinical feature of pneumonia and sepsis. One diabetic patient with a history of coronary artery bypass grafting surgery (CABG) developed subacute lower-limb ischemia during the in-hospital period. This patient underwent femoro-femoral bypass surgery fifty-five days after the primary procedure and in the two-year follow-up had no problem. One of the patients in this group with a previous history of CABG experienced late (four month) acute limb ischemia in the left lower limb. This patient underwent interventional thrombectomy and balloon angioplasty four months after the primary procedure. Also, in the six-month follow-up,another patient suffered from severe type Ib endoleak and pseudoaneurysm formation. This patient was re-hospitalized and subjected to re-stenting six months after the primary intervention. Forty days after the second procedure, this patient had abdominal pain and underwent surgical operation due to aortic aneurysm rupture, but unfortunately expired due to cardiac arrest during surgery. Finally, four patients died during the follow-up period because of nonprocedure-related reasons.

2) In the endovascular repair of thoracic aortic aneurysm (TEVAR) group, endoleaks occurred in none of the patients in the follow-up period. Only one patient who was a heavy smoker developed mild hemoptysis that re-evaluated and there was no problem in his CT scan and CT angiography. This patient, however, expired at home a few months later with the clinical feature like this complication.

3) In the endovascular repair of aortic dissection group, a 67-year-old hypertensive female had the clinical evidence of dissection expansion from the re-entrance about two 
Haji Zeinali AM et al.

months after the endovascular repair. She was not a suitable case for re-intervention or surgery and expired at home following severe chest pain. Another patient, a 74-year-old female, who had prolonged fever with a negative blood culture, with the probable diagnosis of acute arteritis was treated with intravenous antibiotics. Six months after the treatment, she suffered severe chest pain at home and expired with an unknown cause. A 35-year-old man with the diagnosis of Marfan syndrome and a history of bental surgery underwent thoracoabdominal surgical aortic repair due to stent-related edge dissection about twentytwo months after the primary procedure. The procedure was successful and with no complications. A 36-year-old woman underwent re-stenting with the diagnosis of re-dissection six months after TEVAR and had no complications within follow-up.

\subsection{Follow-Up Survival of EVAR Patients}

From the beginning of EVAR in 2006 to $2009,100 \%$ of the patients who were treated by our team had been followed up for an average of thirteen months in the group with endovascular repair of abdominal aortic aneurysm, for an average of 18 months in the group with endovascular repair of thoracic aortic aneurysm, and for an average of 15 months in the group with endovascular repair of aortic dissection. Totally, nine patients died during this followup. The cumulative rate of death was $16.7 \%$ (six cases) in the abdominal aortic aneurysm group (four patients by non-cardiac causes and only two patients by aneurysmrelated causes), $14.3 \%$ (one case) in the thoracic aortic aneurysm group (following severe hemoptysis), and $25.0 \%$ (two cases) in the aortic dissection group (one following an expansion of the dissection from re-entrance and the second one, with the clinical feature of chest pain and unknown exact reason). The severe event-free survival rates were $80.7 \%$ for endovascular repair of abdominal aortic aneurysm, $85.7 \%$ for endovascular repair of thoracic aortic aneurysm, and $65.6 \%$ for endovascular repair of aortic dissection (Figure 2).

\begin{tabular}{|c|c|c|c|}
\hline Clinical Data & $\operatorname{AAA}$ Group $(n=36)$ & TAA Group $(\mathbf{n}=7)$ & TAD Group $(n=8)$ \\
\hline Symptomatic patients & $14(38.9)$ & $4(57.1)$ & $8(100)$ \\
\hline Length of hospital stay, day & $7.36 \pm 4.81$ & $8.00 \pm 3.96$ & $13.25 \pm 9.27$ \\
\hline Length of ICU stay, day & $3.08 \pm 5.01$ & 2.291.38 & $3.75 \pm 5.00$ \\
\hline Creatinine rise $>\mathbf{3 0} \%$ & $4(11.1)$ & 0 & 0 \\
\hline Hemoglobin reduction $>2.5, \mathrm{~g} / \mathrm{dL}$ & $12(33.3)$ & $1(14.3)$ & $3(37.5)$ \\
\hline In-hospital morbidity & $4(11.1)$ & $1(14.3)$ & $2(25)$ \\
\hline In-hospital mortality & $1(2.8)$ & 0 & 0 \\
\hline Mid-term morbidity & $8(22.2)$ & $1(14.3)$ & $6(75.0)$ \\
\hline Survival rate & $29(80.7)$ & $6(85.7)$ & $5(65.6)$ \\
\hline
\end{tabular}

a Abbreviation: AAA, abdominal aortic aneurysm; TAA, thoracic aortic aneurysm; TAD, thoracic aortic dissection.

$\mathrm{b}$ Values are presented as No.(\%) or mean \pm SD.

\section{A}

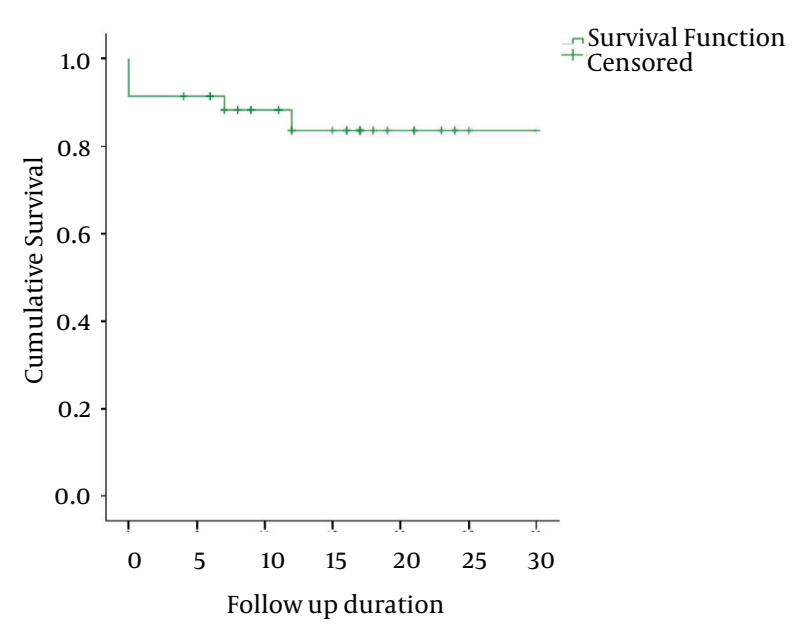

\section{B}

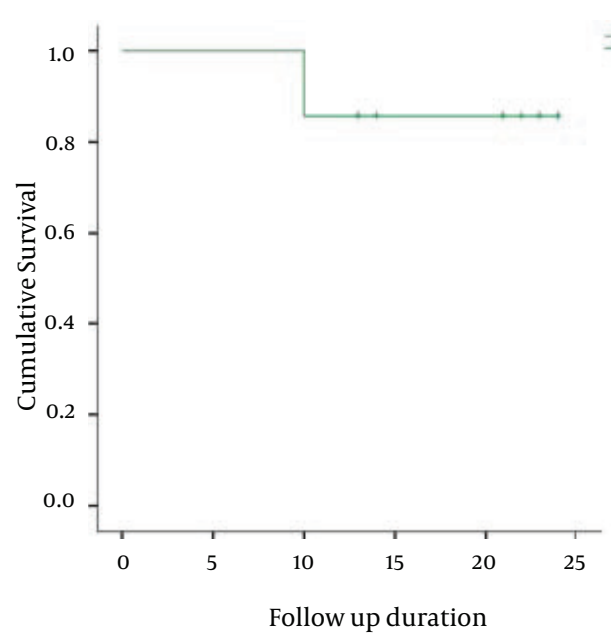

Figure 2. Survival analysis according to Kaplan-Meier curves in EVAR AAA (A) and TEVAR TAA (B) groups 


\section{Discussion}

Since the first endovascular repair of aortic disorders, many vascular surgery centers have selected this procedure for treating thoracoabdominal aortic aneurysms as well as aortic dissections, where its application has contributed to reduction in postoperative poor outcome (15). In the present report, for the first time, we described our clinical experiences in endovascular repair of aortic disorders at Tehran Heart Center, a major referral heart center in Iran and the Middle East. Based on our descriptive findings, the endovascular stent-graft repair of both abdominal and thoracic aortic aneurysms was achieved with high technical success rates $(100 \%$ in the first 51 cases) and low in-hospital and short-term rates of early mortality (2.8\% in AAA and 0.0\%in TAA), low early morbidity, and relatively acceptable hospital stay. In this regard, our findings confirmed the reports of previous surveys reporting procedural success rates higher than $90 \%$ and acceptable patient outcomes (16-20). Although the midterm survival rate in our patients in the different subgroups was acceptable, it has been shown that the early benefits of this procedure are not sustained at follow-up where the mortality is comparable to that of open repair but at an increased cost. Most similar studies have reported early mortality rates of 5\% - 8\% (21-25). In another report, it has been suggested that the early death rate directly depends on the risk stratification, so that the thirtyday mortality rate in large stent-graft series ranges from $0.7 \%$ in low-risk populations to $15.7 \%$ in high-risk patients (26). Nonetheless, our results are similar to those of two recently reported retrospective, controlled studies studying abdominal aneurysm endovascular repair, in which the one-year survival rate after endovascular repair was $95 \%$ and the respective two-year survival rate was approximately $89 \%$. These rates are very close to our findings (27, $28)$. The rate of complication-free survival was lower in some other studies (65.5\% at two years) $(29,30)$.

In our study, five patients in the AAA EVAR group experienced re-intervention with an incidence rate of $13.9 \%$ and two patients in aortic dissection group with an incidence rate of $37.5 \%$. Nevertheless, re-intervention was not observed in the group with thoracic aortic aneurysm. It is worthy of note that re-intervention following open surgery can appear comparable with that seen with elective endovascular repair $(30,31)$, but late re-interventions related to abdominal aortic aneurysms can be more common after endovascular repair (32). These reoperations are usually performed following the appearance of procedural complications such as stent-graft migration, endoleaks, total occlusion of the stent graft, and structural deteriorations that may give rise to re-enlargement of the aortic aneurysm with subsequent catastrophic sequelae (32-35). The most common cause of re-intervention is the endoleak. Some authors believe that repairing any leak persisting for more than three months after the endovascular treatment of an aortic aneurysm is necessary (36).
With respect to management of aortic dissection by endovascular repair in our participants, serious procedural complications appeared more often in these patients than in those who suffered from aortic aneurysms, and the former group had a notably lower mid-term survival rate than the other groups.

We herein reported the short- and mid to long-term results of the stent-graft technique in patients with aortic aneurysms or dissections amongst the Iranian population. Still, a thorough long-term evaluation of the outcomes in terms of cost and quality of life for these patients is recommended.

Endovascular stent-graft repair of abdominal and thoracic aortic aneurysms as well as aortic dissection was achieved with high technical success rates in tandem with low rates of early mortality and early morbidity, acceptable hospital stay, and acceptable mid-term survival rate among our Iranian patients. Symptom-free survival is better in aortic aneurysm EVAR than aortic dissection. EVAR is the acceptable treatment especially in inoperable patients who have other comorbidities.

\section{Authors' Contributions}

Study concept and design: Ali Mohammad Haji Zeinali. Acquisition of data: Roshanak Mahmoodian. Analysis and interpretation of data: Ali Mohammad Haji Zeinali. Drafting of the manuscript: Ali Mohammad Haji Zeinali, Roshanak Mahmoodian, and Masoumeh Lotfi-Tokaldany. Statistical analysis: Mehrdad Sheikhvatan and Masoumeh Lotfi-Tokaldany. Administrative technical and material support: Ali Mohammad Haji Zeinali, Mehrab Marzban, Mohammadreza Zafarghandi, Mahmood Shirzad, and Shapour Shirani.

\section{Funding/Support}

No funding and support was received.

\section{References}

1. Evar trial participants. Endovascular aneurysm repair and outcome in patients unfit for open repair of abdominal aortic aneurysm (EVAR trial 2): randomised controlled trial. Lancet. 2005;365(9478):2187-92.

2. Fann JI, Miller DC. Endovascular treatment of descending thoracic aortic aneurysms and dissections. Surg Clin North Am. 1999;79(3):551-74.

3. Haji-Zeinali AM, Ghazi P, Alidoosti M. Self-expanding nitinol stent implantation for treatment of aortic coarctation.J Endovasc Ther. 2009;16(2):224-32.

4. Criado FJ, Abul-Khoudoud OR, Domer GS, McKendrick C, Zuzga M, Clark NS, et al. Endovascular repair of the thoracic aorta: lessons learned. Ann Thorac Surg. 2005;80(3):857-63.

5. Ghazi P, Haji-Zeinali AM, Shafiee N, Qureshi SA. Endovascular ab dominal aortic stenosis treatment with the OptiMed self-expandable nitinol stent. Catheter Cardiovasc Interv. 2009;74(4):634-41.

6. Chahwan S, Comerota AJ, Pigott JP, Scheuermann BW, Burrow J Wojnarowski D. Elective treatment of abdominal aortic aneurysm with endovascular or open repair: the first decade. J Vasc Surg. 2007; 45(2):258-62. 


\section{Haji Zeinali AM et al.}

7. Brown LC, Brown EA, Greenhalgh RM, Powell JT, Thompson SG. UK EVAR trial participants. (EVAR trial 1) Lancet. Biosurg Surg Technol. 2004;364:843-8.

8. Prinssen M, Verhoeven EL, Buth J, Cuypers PW, van Sambeek MR, Balm R, et al. A randomized trial comparing conventional and endovascular repair of abdominal aortic aneurysms. $N$ Engl J Med. 2004;351(16):1607-18.

9. Ghazi P, Haji-Zeinali AM. Transcatheter closure of a patent arterial duct with the Amplatzer duct occluder in the area of a large thoracic aortic aneurysm. Cardiol Young. 2009;19(2):209-11.

10. Ghazi P, Haji-Zeinali AM, Ghasemi M, Pour MZ. Transcatheter stent implantation for the treatment of abdominal aortic coarctation and right renal artery stenosis in takayasu's arteritis: a case with a 4-year follow up. Hellenic J Cardiol. 2011;52(2):182-5.

11. Ghazi P, Haji-Zeinali AM, Zarghampour M. Staged endovascular treatment of left common carotid artery large aneurysm with Gore-Hemobahn stent-graft after right common carotid artery stenosis angioplasty. J Invasive Cardiol. 2010;22(7):E129-31.

12. Patel VI, Conrad MF, Kwolek CJ, Ouriel K, Fairman RM, Cambria RP. Propensity-matched cohort validates findings of the VALOR trial. J Vasc Surg. 2011;54(1):22-9.

13. Heikkinen MA, Arko FR, Zarins CK. What is the significance of endoleaks and endotension. Surg Clin North Am. 2004;84(5):1337-52.

14. Scott RA, Wilson NM, Ashton HA, Kay DN. Influence of screening on the incidence of ruptured abdominal aortic aneurysm: 5-year results of a randomized controlled study. Br J Surg. 1995;82(8):1066-70.

15. Dake MD, Kato N, Mitchell RS, Semba CP, Razavi MK, Shimono T, et al. Endovascular stent-graft placement for the treatment of acute aortic dissection. N Engl J Med.1999;340(20):1546-52.

16. Nienaber CA, Fattori R, Lund G, Dieckmann C, Wolf W, von Kodolitsch Y, et al. Nonsurgical reconstruction of thoracic aortic dissection by stent-graft placement. NEnglJ Med.1999;340(20):1539-45.

17. Bortone AS, Schena S, D'Agostino D, Dialetto G, Paradiso V, Mannatrizio $G$, et al. Immediate versus delayed endovascular treatment of post-traumatic aortic pseudoaneurysms and type B dissections: retrospective analysis and premises to the upcoming European trial. Circulation. 2002;106(12 Suppl 1):I234-40.

18. Kato N, Hirano T, Ishida M, Shimono T, Cheng SH, Yada I, et al. Acute and contained rupture of the descending thoracic aorta: treatment with endovascular stent grafts. J Vasc Surg. 2003;37(1):100-5.

19. Shimono T, Kato N, Yasuda F, Suzuki T, Yuasa U, Onoda K, et al. Transluminal stent-graft placements for the treatments of acute onset and chronic aortic dissections. Circulation. 2002;106(12 Suppl 1):I241-7.

20. Zarins CK, Harris EJ. Operative repair for aortic aneurysms: the gold standard. J Endovasc Surg. 1997;4(3):232-41.

21. Lawrence PF, Gazak C, Bhirangi L, Jones B, Bhirangi K, Oderich G, et al. The epidemiology of surgically repaired aneurysms in the United States.J Vasc Surg. 1999;30(4):632-40.
22. Heller JA, Weinberg A, Arons R, Krishnasastry KV, Lyon RT, Deitch JS, et al. Two decades of abdominal aortic aneurysm repair: have we made any progress? JVasc Surg. 2000;32(6):1091-100.

23. Bayly PJ, Matthews JN, Dobson PM, Price ML, Thomas DG. In-hospital mortality from abdominal aortic surgery in Great Britain and Ireland:Vascular Anaesthesia Society audit. BrJSurg. 2001;88(5):687-92.

24. Huber TS, Wang JG, Derrow AE, Dame DA, Ozaki CK, Zelenock GB, et al. Experience in the United States with intact abdominal aortic aneurysm repair. JVasc Surg. 2001;33(2):304-10.

25. Buth J, Laheij RJ. Early complications and endoleaks after endovascular abdominal aortic aneurysm repair: report of a multicenter study.J Vasc Surg. 2000;31(1 Pt 1):134-46.

26. Goueffic Y, Becquemin JP, Desgranges P, Kobeiter H. Midterm survival after endovascular versus open repair of infrarenal aortic aneurysms. JEndovasc Ther. 2005;12(1):47-57.

27. Cao P, Verzini F, Parlani G, Romano L, De Rango P, Pagliuca V, et al. Clinical effect of abdominal aortic aneurysm endografting: 7-year concurrent comparison with open repair. J Vasc Surg. 2004;40(5):841-8.

28. Blankensteijn JD, de Jong SE, Prinssen M, van der Ham AC, Buth J van Sterkenburg SM, et al. Two-year outcomes after conventional or endovascular repair of abdominal aortic aneurysms. $N$ Engl J Med. 2005;352(23):2398-405.

29. Thomas SM, Beard JD, Ireland M, Ayers S, Vascular Society of Great B, Ireland. et al. Results from the prospective registry of endovascular treatment of abdominal aortic aneurysms (RETA): mid term results to five years. Eur J Vasc Endovasc Surg. 2005;29(6):563-70.

30. Schermerhorn ML, O'Malley AJ, Jhaveri A, Cotterill P, Pomposelli F, Landon BE. Endovascular vs. open repair of abdominal aortic aneurysms in the Medicare population. $N$ Engl $\mathrm{J} \mathrm{Med.}$ 2008;358(5):464-74.

31. BrandtM,Hussel K, Walluscheck KP, Muller-HulsbeckS, Jahnke T, Rahimi A, et al. Stent-graft repair versus open surgery for the descending aorta: a case-control study.J Endovasc Ther. 2004;11(5):535-8.

32. Holzenbein TJ, Kretschmer G, Thurnher S, Schoder M, Aslim E, Lammer J, et al. Midterm durability of abdominal aortic aneurysm endograft repair: a word of caution. J Vasc Surg. 2001;33(2 Suppl):S46-54.

33. Alric P, Hinchliffe RJ, Wenham PW, Whitaker SC, Chuter TA, Hopkinson BR. Lessons learned from the long-term follow-up of a first-generation aortic stent graft.J Vasc Surg. 2003;37(2):367-73.

34. Gorich J, Rilinger N, Sokiranski R, Kramer SC, Ermis C, Schutz A, et al. Treatment of leaks after endovascular repair of aortic aneurysms. Radiology. 2000;215(2):414-20.

35. Zarins CK, Crabtree T, Bloch DA, Arko FR, Ouriel K, White RA. Endovascular aneurysm repair at 5 years: Does aneurysm diameter predict outcome? J Vasc Surg. 2006;44(5):920-9.

36. Erbel R, Alfonso F, Boileau C, Dirsch O, Eber B, Haverich A, et al. Diagnosis and management of aortic dissection. Eur Heart $J$. 2001;22(18):1642-81. 